\title{
ON INTERVAL RECURRENT SUMS OF INDEPENDENT RANDOM VARIABLES
}

\section{FRANK SPITZER}

1. Introduction. This note deals with infinite sequences $\left\{X_{i}\right\}$ of mutually independent identically distributed random variables. Their $n$th partial sums are denoted by $S_{n}=\sum_{i=1}^{n} X_{i}$. In all that follows each sequence $\left\{X_{i}\right\}$ is assumed to satisfy the further conditions:

(A) For some $1 \leqq \alpha \leqq 2$

$$
\lim _{n \rightarrow \infty} \operatorname{Pr}\left\{S_{n} \leqq x n^{1 / \alpha}\right\}=V_{\alpha}(x),
$$

where $V_{\alpha}(x)$ is the distribution function of the symmetric stable law of order $\alpha$ (with the characteristic function $\exp \{-|\lambda| \alpha\}$ ).

(B) The distribution for each $X_{i}, i=1,2, \cdots$, is determined by a probability density $f(x)$, such that $f(x) \in L^{p}(-\infty, \infty)$ for some $p>1$.

Under the above conditions ${ }^{1}$ it was shown by Kallianpur and Robbins [1] that the sequence of partial sums $S_{n}$ is interval recurrent. This means that

$$
\operatorname{Pr}\left\{\left|S_{n}-a\right|<\epsilon \text { for infinitely many } n\right\}=1
$$

for every $a$ and every $\epsilon>0$. If on the other hand (A) holds with $\alpha<1$, then the probability in (1) is zero.

The results of this note take the form of two limit theorems of familiar type. They show that the recurrence behavior of the sequence $\left\{S_{n}\right\}$ depends only on the index $\alpha$ of the domain of attraction of the sequence. Condition (B) excludes from consideration all sequences of random variables of the lattice type for which the theorems below are clearly false. However it seems plausible that the sufficient conditions (A) and (B) may be replaced by far weaker conditions.

THEOREM $1 .{ }^{2}$ Let $\left\{S_{n}\right\}$ satisfy conditions (A) and (B) with exponent $1 \leqq \alpha \leqq 2$. Let

Presented to the Society, December 29, 1954; received by the editors March 21, 1955.

${ }^{1}$ In [1] it is required that $f(x) \in L^{p}(-\infty, \infty)$ for some $1<p \leqq 2$. This condition is satisfied if $f(x) \in L^{p}(-\infty, \infty)$ for some $p>1$ because $f(x) \in L^{1}(-\infty, \infty)$.

2 Actually a far stronger result will be proved, the statement of which is contained in equation (12). 


$$
C(n, \alpha)=\left\{\begin{array}{lr}
\frac{1}{2} \alpha \sin \frac{\pi}{\alpha} n^{1 / \alpha-1} & \text { for } 1<\alpha \leqq 2, \\
\frac{1}{2} \pi(\log n)^{-1} & \text { for } \alpha=1,
\end{array}\right.
$$

and let $E_{p}(z)$ denote the Mittag-Leffler function

$$
E_{p}(z)=\sum_{n=0}^{\infty} \frac{z^{n}}{\Gamma(1+p n)} .
$$

Then the limiting distribution of $\min _{1 \leqq k \leqq n}\left|S_{k}-a\right|$, for any $-\infty<a$ $<\infty$, normalized by the appropriate $C(n, \alpha)$, is given by

$\lim _{n \rightarrow \infty} \operatorname{Pr}\left\{\min _{1 \leqq k \leqq n}\left|S_{k}-a\right| \leqq x C(n, \alpha)\right\}= \begin{cases}0 & \text { for } x \leqq 0, \\ 1-E_{1-1 / \alpha}(-x) & \text { for } x \geqq 0 .\end{cases}$

Theorem 1 has a simple form when $\left\{S_{n}\right\}$ satisfies (A) and (B) with $\alpha=1$ or $\alpha=2$. When $\alpha=1, x \geqq 0$,

$$
\lim _{n \rightarrow \infty} \operatorname{Pr}\left\{\min _{1 \leqq k \leqq n}\left|S_{k}-a\right| \leqq \frac{\pi}{2 \log n} x\right\}=\frac{x}{1+x} .
$$

When $\alpha=2, x \geqq 0$,

$$
\lim _{n \rightarrow \infty} \operatorname{Pr}\left\{\min _{1 \leqq k \leqq n}\left|S_{k}-a\right| \leqq \frac{x}{n^{1 / 2}}\right\}=1-\frac{2}{\pi^{1 / 2}} e^{x^{2}} \int_{x}^{\infty} e^{-t^{2}} d t .
$$

Note that if (B) holds and if the random variables $X_{i}$ have mean zero and finite variance $\sigma^{2}$, then the sequence $S_{n}=\left(2^{1 / 2} / \sigma\right) \sum_{i=1}^{n} X_{i}$ satisfies the conditions of Theorem 1 with $\alpha=2$.

The strong law corresponding to Theorem 1 is

THEOREM 2. With $\left\{S_{n}\right\}$ as in Theorem 1 , and $\left\{a_{n}\right\}$ a nonincreasing positive sequence, the probability that $\left|S_{n}-a\right| \leqq n^{1 / \alpha} a_{n}$ for infinitely many values of $n$ is zero or one according as $\sum_{n=1}^{\infty} a_{n}$ converges or diverges.

2. Proof of Theorem 1. For both theorems the following result due to Kallianpur and Robbins [1, Lemma 6.1] is basic.

"Under the conditions (A) and (B) ${ }^{3}$ the density $f_{n}(x)$ of $S_{n}$ is, for large enough $n$, of the form

$$
f_{n}(x)=\frac{1}{\pi \alpha} \Gamma\left(\frac{1}{\alpha}\right) n^{-1 / \alpha}+c_{n} g_{n}(x),
$$

where $c_{n}=o\left(n^{-1 / \alpha}\right)$ and $\left|g_{n}(x)\right|<M(I)$ for all $x$ in any finite interval $I . "$

See footnote 1. 
To prove Theorem 1 we define the events $E_{\mathbf{k}}^{n}(x), 1 \leqq k \leqq n$, in the sample space of the sequence $\left\{X_{i}\right\}$ as

$$
E_{k}^{n}(x)=\left\{w|| S_{k}-a \mid \leqq x C(n, \alpha)\right\} .
$$

The random variables $I_{k}^{n}(\omega, x)=1$ or 0 according as $\omega \in E_{k}^{n}$ or not are used to define the random variables

$$
N_{n}(x)=N_{n}(\omega, x)=\sum_{k=1}^{n} I_{k}^{n}(\omega, x) .
$$

By a well-known combinatorial formula to be found in the book of Feller [2]

$$
p_{\nu}(x, n)=\operatorname{Pr}\left\{N_{n}(x)=\nu\right\}=\sum_{k=\nu}^{n}(-1)^{k-\nu}\left(\begin{array}{l}
k \\
\nu
\end{array}\right) \rho_{k}(n, x),
$$

where

$$
\rho_{k}(n, x)=\sum_{1 \leqq i_{1}<\cdots<i_{k} \leqq n} \operatorname{Pr}\left[\bigcap_{s=1}^{k} E_{i_{g}}^{n}(x)\right], \quad \rho_{0}(n, x)=1 .
$$

Now it may be seen that Theorem 1 is equivalent to the statement

$$
\lim _{n \rightarrow \infty} p_{0}(x, n)=E_{1-1 / \alpha}(-x) .
$$

To prove (6) it must first be shown that

$$
\rho_{k}(x)=\lim _{n \rightarrow \infty} \rho_{k}(n, x)=\frac{x^{k}}{\Gamma[1+k(1-1 / \alpha)]} .
$$

If $f_{n}(x)$ is the density of $S_{n}$, and if $\Omega_{k}(n, x)$ is the region in Euclidean $k$-space defined by

$$
\left|\sum_{i=1}^{\infty} x_{i}-a\right| \leqq x C(n, \alpha), \quad s=1,2, \cdots, k,
$$

then, according to (3) and (5),

$$
\begin{aligned}
\rho_{k}(n, x) & =\sum_{1 \leqq i_{1}<\cdots<i_{k} \leqq n} \int_{\Omega_{k}(n, x)} \ldots \int f_{i_{1}}\left(x_{1}\right) f_{i_{2}-i_{1}}\left(x_{2}\right) \cdots \\
& =\sum_{i_{\nu} \geqq 1, j_{1}+\cdots+j_{k} \leqq n} \int_{\Omega_{k}(n, x)} \ldots \int \prod_{i_{i_{k}-i_{k-1}}\left(x_{k}\right) d x_{1} \cdots d x_{k} .}^{k} f_{i_{\nu}}\left(x_{\nu}\right) d x .
\end{aligned}
$$

We may assume that (2) holds with $n \geqq N$ for our given sequence $\left\{X_{i}\right\}$. For the purpose of using (2) to prove (7) one can set 


$$
\sigma_{k}(n, x) \leqq \rho_{k}(n, x) \leqq \sigma_{k}(n, x)+\delta_{k}(n, x)
$$

with

$$
\sigma_{k}(n, x)=\sum_{j_{\nu} \geqq N, j_{1}+\cdots+j_{k} \leqq n} \int_{\Omega_{k}(n, x)} \ldots \int \prod_{\nu=1}^{k} f_{j_{\nu}}\left(x_{\nu}\right) d x_{\nu}
$$

We note that for some $A_{k}>0$, independent of $n$, each region $\Omega_{k}(n, x)$ is contained in the region

$$
\left|x_{i}\right| \leqq A_{k} C(n, \alpha), \quad i=1,2, \cdots, k .
$$

Hence one may take

$$
\prod_{\nu=1}^{k} \delta_{\nu} \delta_{k}(n, x)=\sum_{1 \leqq \min _{1} \varliminf_{\nu} k_{k}<N, j_{1}+j_{2}+\cdots+j_{k} \leqq n} \prod_{\nu=1}^{k} \delta_{\nu},
$$

with

$$
\delta_{\nu}=\int_{-A_{k} C(n, \alpha)}^{\Delta_{k} C(n, \alpha)} f_{j_{\nu}}(x) d x \text { or } 1 \text { according as } j_{\nu} \geqq N \text { or } j_{\nu}<N .
$$

The volume of $\Omega_{k}(n, x)$ is $[2 x C(n, \alpha)]^{k}$ and it follows from (2) that, as $n \rightarrow \infty$,

$$
\begin{aligned}
\sigma_{k}(n, x)= & {\left[2 x C(n, \alpha) \cdot \frac{1}{\pi \alpha} \Gamma\left(\frac{1}{\alpha}\right)\right]^{k} } \\
& \cdot[1+o(1)] \sum_{j_{\nu} \geqq N, j_{1}+\cdots+j_{k} \leqq n}\left[j_{1} j_{2} \cdots j_{k}\right]^{-1 / \alpha},
\end{aligned}
$$

where

$$
2 x C(n, \alpha) \cdot \frac{1}{\pi \alpha} \Gamma\left(\frac{1}{\alpha}\right)=\frac{x \cdot n^{1 / \alpha-1}}{\Gamma(1-1 / \alpha)} \quad \text { if } 1<\alpha \leqq 2,
$$

and

$$
x / \log n \quad \text { if } \alpha=1 .
$$

It is clear that the limit of $\sigma_{k}(n, x)$ as $n \rightarrow \infty$ does not depend on the value of $N$, and a calculation which is similar to those carried out in [1] and [3] yields

$$
\lim _{n \rightarrow \infty} \sigma_{k}(n, x)=\frac{x^{k}}{\Gamma[1+k(1-1 / \alpha)]} .
$$

Straightforward calculation also shows that $\delta_{k}(n, x) \rightarrow 0$ as $n \rightarrow \infty$, and in view of (8) the proof of (7) is now complete.

To prove (6), and thus Theorem 1, it would suffice to show directly that 


$$
\begin{aligned}
\lim _{n \rightarrow \infty} p_{0}(x, n) & =\lim _{n \rightarrow \infty} \sum_{k=0}^{n}(-1)^{k} \rho_{k}(n, x) \\
& =\sum_{k=0}^{\infty}(-1)^{k} \lim _{n \rightarrow \infty} \rho_{k}(n, x)=E_{1-1 / \alpha}(-x) .
\end{aligned}
$$

However, it seems impossible to justify the interchange of limits. The method used instead is longer but has the advantage of yielding a stronger result.

Equation (4) implies that the $k$ th moment of $N_{n}(x)$ is

$$
E\left\{\left[N_{n}(x)\right]^{k}\right\}=\sum_{\nu=0}^{n} p_{\nu}(x, n) \nu^{k}=\sum_{\nu=0}^{n} \rho_{\nu}(n, x) V_{\nu}(k),
$$

where

$$
V_{\nu}(k)=\sum_{\mu=0}^{\nu}\left(\begin{array}{l}
\nu \\
\mu
\end{array}\right)(-1)^{\nu-\mu} \mu^{k} .
$$

Thus $(1 / k !) V_{\nu}(k)$ is the coefficient of $x^{k}$ in the power series expansion of $\left(e^{x}-1\right)^{\prime}$. Hence $V_{\nu}(k)=0$ for $\nu>k$, so that $E\left\{\left[N_{n}(x)\right]^{k}\right\}$ is a finite sum of $k+1$ terms for any value of $n$. Hence

$$
m_{k}=\lim _{n \rightarrow \infty} E\left\{\left[N_{n}(x)\right]^{k}\right\}=\sum_{\nu=0}^{k} \rho_{\nu}(x) V_{\nu}(k),
$$

with $\rho_{\nu}(x)$ given in equation (7). Let $\phi_{n}(\lambda)$ be the characteristic function of $N_{n}(x)$, and define, using (7), (9), and (10),

$$
\begin{aligned}
\phi(\lambda) & =\sum_{k=0}^{\infty} \frac{(i \lambda)^{k}}{k !} m_{k}=\sum_{k=0}^{\infty} \frac{(i \lambda)^{k}}{k !} \sum_{\nu=0}^{k} \rho_{\nu}(x) V_{\nu}(k) \\
& =\sum_{\nu=0}^{\infty} \rho_{\nu}(x)\left(e^{i \lambda}-1\right)^{\nu}=E_{1-1 / \alpha}\left(x e^{i \lambda}-x\right) .
\end{aligned}
$$

$\phi(\lambda)$ is seen to be analytic in a neighborhood of the origin for every $x>0$ and for every $1 \leqq \alpha \leqq 2$. Hence the moment problem defined by equation (10) has a unique solution, and the sequence $\phi_{n}(\lambda)$ converges to the characteristic function $\phi(\lambda)$. Since $\phi(\lambda)$ is periodic with period $2 \pi$ for real $\lambda$, it is the characteristic function of a distribution function which is constant except for jumps $p_{k}(x)$ at the points $k=0,1, \cdots$. But as $\phi_{n}(\lambda) \rightarrow \phi(\lambda)$ we must have

$$
p_{k}(x)=\lim _{n \rightarrow \infty} p_{k}(x, n) .
$$

Moreover, for $|t| \leqq 1$, equation (11) implies 


$$
\sum_{k=0}^{\infty} p_{k}(x) t^{k}=\lim _{n \rightarrow \infty} E\left[t^{N_{n}(x)}\right]=E_{1-1 / \alpha}(x t-x) .
$$

Letting $t$ tend to zero in (12) we have

$$
p_{0}(x)=\lim _{n \rightarrow \infty} \operatorname{Pr}\left\{N_{n}(x)=0\right\}=E_{1-1 / \alpha}(-x),
$$

so that Theorem 1 has been established as a special case of the result of equation (12).

3. Proof of Theorem 2. We shall assume that $a=0$, the method of proof being the same for any value of $a$. Let $E_{n}=\left\{\omega \mid S_{n} \in I_{n}\right\}$ where $I_{n}$ is the interval $\left(-n^{1 / \alpha} a_{n}, n^{1 / \alpha} a_{n}\right)$. First assume that $\sum_{n=1}^{\infty} a_{n}<\infty$. Then $n^{1 / \alpha} a_{n} \rightarrow 0$ as $n \rightarrow \infty$, and one obtains from (2) that

$$
\begin{aligned}
\operatorname{Pr}\left\{E_{n}\right\} & =\int_{I_{n}} f_{n}(x) d x=2 n^{1 / \alpha} a_{n}\left[\frac{1}{\pi \alpha} \Gamma\left(\frac{1}{\alpha}\right) n^{-1 / \alpha}+o\left(n^{-1 / \alpha}\right)\right] \\
& =A a_{n}+o\left(a_{n}\right) \text { as } n \rightarrow \infty, \text { with } A=\frac{2}{\pi \alpha} \Gamma\left(\frac{1}{\alpha}\right) .
\end{aligned}
$$

Hence $\sum_{n=1}^{\infty} \operatorname{Pr}\left\{E_{n}\right\}<\infty$, and by the Borel-Cantelli lemma

$$
\operatorname{Pr}\left\{E_{n} \text { for infinitely many } n\right\}=0 \text {, }
$$

which proves the first part of Theorem 2.

To prove the second part of Theorem 2 one has to assume that $\sum_{n=1}^{\infty} a_{n}=\infty$. In fact, it will clearly be sufficient to consider only the case when, in addition to $\sum_{n=1}^{\infty} a_{n}=\infty$,

$$
a_{n} \leqq[n \log n]^{-1} \quad \text { for large enough values of } n \text {. }
$$

Just as before, one shows that $\sum_{n=1}^{\infty} \operatorname{Pr}\left\{E_{n}\right\}=\infty$. The events $E_{n}$ are not independent, but to conclude that

$$
\operatorname{Pr}\left\{E_{n} \text { for infinitely many } n\right\}=1
$$

it will suffice, according to Chung and Erdös [4], to verify that the sequence $\left\{E_{n}\right\}$ satisfies the following three conditions.

(I) For any positive integers $n$ and $h, n \geqq h$, there exist positive functions $c(h)$ and $H(n, h)>h$ such that for every $k>H(n, h)$

$$
\operatorname{Pr}\left\{E_{k} \mid E_{h}^{\prime} \cap E_{h+1}^{\prime} \cap \cdots \cap E_{n}^{\prime}\right\}>c(h) \operatorname{Pr}\left\{E_{k}\right\} .
$$

(Primes denote the complements.)

(II) A set of events $\left\{E_{n_{i}}\right\}, i=1,2, \cdots, s(n)$ can be found for each value of $n$ with the property

$$
\sum_{i=1}^{8(n)} \operatorname{Pr}\left\{E_{n_{i}} \mid E_{n}\right\}<c_{1},
$$


where $c_{1}$ is a positive constant independent of $n$.

(III) There is a constant $c_{2}>0$, such that

$$
\operatorname{Pr}\left\{E_{k} \mid E_{n}\right\}<c_{2} \operatorname{Pr}\left\{E_{k}\right\} \text { for all } n,
$$

whenever $k>n$ and $E_{k}$ is not in the set $\left\{E_{n_{i}}\right\}$ chosen in (II) to correspond to $E_{n}$.

With $E_{k}$ and the interval $I_{k}$ as defined above,

$$
\operatorname{Pr}\left\{E_{k} \mid E_{h}^{\prime} \cap \cdots \cap E_{n}^{\prime}\right\}=\int_{I_{k}} \int_{-\infty}^{\infty} f_{k-n}(x-y) d G_{n, h}(y) d x,
$$

where $G_{n, h}(x)$ is the conditional probability distribution

$$
G_{n, h}(x)=\operatorname{Pr}\left\{S_{n} \leqq x \mid E_{h}^{\prime} \cap \cdots \cap E_{n}^{\prime}\right\} .
$$

Let $I=I(n, h)$ be a bounded interval with the property that

$$
\int_{I} d G_{n, h}(x)=\frac{1}{2} .
$$

Then equation (2) applied to (15) implies

$$
\begin{aligned}
\operatorname{Pr}\left\{E_{k} \mid E_{h}^{\prime} \cap \cdots \cap E_{n}^{\prime}\right\} & \geqq \int_{I_{k}} \int_{I} f_{k-n}(x-y) d G_{n, h}(y) d x \\
& =\frac{1}{2} A a_{k}+o\left(a_{k}\right),
\end{aligned}
$$

where $A$ is the constant defined in (13). Combining (16) and (13) one obtains

(17) $\operatorname{Pr}\left\{E_{k} \mid E_{h}^{\prime} \cap \cdots \cap E_{n}^{\prime}\right\} \geqq \operatorname{Pr}\left\{E_{k}\right\} \cdot[1 / 2+o(1)]$, as $k \rightarrow \infty$, so that condition (I) is satisfied with $c(h)=1 / 3$.

To verify condition (II), let the set $\left\{E_{n_{i}}\right\}$ which corresponds to $E_{n}$ consist of the events $E_{n+1}, E_{n+2}, \cdots, E_{2 n}$. To show that the sequence of sums $\sum_{k=1}^{n} \operatorname{Pr}\left\{E_{n+k} \mid E_{n}\right\}$ is bounded, one can write

$$
\operatorname{Pr}\left\{E_{n+k} \mid E_{n}\right\}=\int_{I_{n+k}} \int_{I_{n}} f_{k}(x-y) d H_{n}(y) d x
$$

where

$$
H_{n}(x)=\operatorname{Pr}\left\{S_{n} \leqq x \mid S_{n} \in I_{n}\right\} .
$$

Equation (2) applied to (18) now shows that, as $k \rightarrow \infty$, (19) $\operatorname{Pr}\left\{E_{n+k} \mid E_{n}\right\}=A(n+k)^{1 / \alpha} k^{-1 / \alpha} a_{n+k}+(n+k)^{1 / \alpha} a_{n+k} o\left(k^{-1 / \alpha}\right)$. Using (14), the boundedness of the sequence $\sum_{k=1}^{n} \operatorname{Pr}\left\{E_{n+k} \mid E_{n}\right\}$ is 
a consequence of the existence of the limit

$$
\lim _{n \rightarrow \infty} \sum_{k=1}^{n} \frac{(n+k)^{1 / \alpha-1} k^{-1 / \alpha}}{\log (n+k)}<\infty, \quad \text { for } 1 \leqq \alpha \leqq 2 .
$$

Finally, to verify condition (III), we must show that the ratios

$$
\frac{\operatorname{Pr}\left\{E_{n} \mid E_{k}\right\}}{\operatorname{Pr}\left\{E_{n}\right\}}
$$

are bounded for all $n>2 k$. For large enough $k,(19)$ and (13) combined yield

$$
\frac{\operatorname{Pr}\left\{E_{n} \mid E_{k}\right\}}{\operatorname{Pr}\left\{E_{n}\right\}}=\left(\frac{k}{n}\right)^{-1 / \alpha}+o(1) \leqq 2^{-1 / \alpha}+o(1),
$$

so that (III) holds, and the proof of Theorem 2 is complete.

\section{BIBLIOGRAPHY}

1. G. Kallianpur and $\mathrm{H}$. Robbins, The sequence of sums of independent random variables, Duke Math. J. vol. 21 (1954) pp. 285-307.

2. W. Feller, An introduction to probability theory and its applications, vol. 1, New York, Wiley, 1950.

3. K. L. Chung and M. Kac, Remarks on fluctuations of sums of independent random variables, Memoirs of the American Mathematical Society, vol. 6, 1951.

4. K. L. Chung and P. Erdös, On the application of the Borel-Cantelli lemma, Trans. Amer. Math. Soc. vol. 72 (1952) pp. 179-186.

California Institute of Technology 